\title{
Allogeneic stem cell transplantation using alemtuzumab-containing regimens in severe aplastic anemia
}

\author{
S. Gandhi • A. G. Kulasekararaj · G. J. Mufti • \\ J. C. W. Marsh
}

Received: 8 April 2013/Accepted: 10 April 2013/Published online: 30 April 2013

(C) The Japanese Society of Hematology 2013

\begin{abstract}
Alemtuzumab, a humanized anti-CD52, IgG1 monoclonal antibody, is used to reduce graft-versus- host disease (GVHD) and aid engraftment after allogeneic haemopoietic stem cell transplant (HSCT). Its associated low incidence of GVHD makes it an attractive alternative to anti-thymocyte globulin (ATG) in transplant conditioning regimen for severe aplastic anaemia (SAA). We have reviewed the use of alemtuzumab-based conditioning regimen for HSCT in SAA and show that it results in sustained haematological engraftment, a very low incidence of chronic GVHD without an increase in viral infections. Intriguingly, alemtuzumab appears to induce tolerance post-HSCT with the findings of stable mixed $\mathrm{T}$ cell chimerism with full donor myeloid chimerism and the absence of chronic GVHD, and which persist on withdrawal of post-graft immunosuppression. Finally, its low toxicity profile may permit future application of HSCT to older patients with SAA who fail to respond to immunosuppressive therapy.
\end{abstract}

Keywords Alemtuzumab - Aplastic anemia . Haemopoietic stem cell transplant (HSCT)

\section{Introduction}

Allogeneic haemopoietic stem cell transplantation (HSCT) is the only potentially curative therapy for patients with acquired severe aplastic anaemia (SAA). HSCT is the

S. Gandhi · A. G. Kulasekararaj · G. J. Mufti ·

J. C. W. Marsh ( $\square)$

Department of Haematological Medicine, King's College

Hospital/King's College London, Denmark Hill,

London SE5 9RS, UK

e-mail: judith.marsh@nhs.net initial treatment of choice for newly diagnosed patients up to the age of 40-50 years in the presence of an HLAmatched sibling donor (MSD). Matched unrelated donor (MUD) HSCT is indicated after failure to respond to a course of immunosuppressive therapy (IST) with antithymocyte globulin (ATG) and cyclosporin (CSA) [1, 2].

Survival has significantly improved over the past 3 decades, because of the introduction of CSA in the early 1980s, resulting in reduced graft rejection, and better supportive care with transplant regimens resulting in lower transplant related mortality. Improved HLA matching and better conditioning regimens have also contributed to improved outcomes after MUD HSCT. Long-term survival is seen in 80-90\% of patients following MSD HSCT [3-6] and $75-80 \%$ for MUD HSCT in SAA [7-10].

However, the overall survival (OS) after HSCT for SAA is age-dependent, with worse outcomes in older patients [11, 12], and better outcomes in children [13-15]. Graft-versushost disease (GVHD) also predicts for worse survival after HSCT in SAA [5]. There is no advantage for any degree of GVHD in SAA, in contrast to the beneficial impact of graftversus leukaemia (GVL) effect in myeloid malignancies. The historical use of TBI-based conditioning regimens reduced the risk of graft rejection, but increased GVHD, pneumonitis and serious long-term effects, such as second malignancies and reduced growth and development in children $[16,17]$. ATG-based conditioning regimens are now most commonly used, to reduce GVHD and aid engraftment; for MSD HSCT; ATG is used with high dose cyclophosphamide (CY) $200 \mathrm{mg} / \mathrm{kg}$ for patients $<30-40$ years old, and for MUD HSCT, with fludarabine, and lower dose CY with or without low dose TBI (2 Gy). A recent retrospective EBMT study of 1,886 patients showed that the addition of ATG improved OS after MSD HSCT [6]; a previous prospective randomized study from the CIBMTR showed no 
significant difference in OS when ATG was added to CY $200 \mathrm{mg} / \mathrm{kg}$, but that study was underpowered due to relatively low number of patients enrolled [4]. However, ATGbased conditioning is associated with up to $30 \%$ incidence of chronic GVHD [3-6], and severe chronic GVHD impacts quality of life as well as OS (Table 1).

\section{Alemtuzumab: the humanized form of CAMPATH-1 antibodies}

The CAMPATH-1 monoclonal antibodies recognise CD52, a GPI-linked membrane protein, which is expressed on peripheral blood $\mathrm{T}$ and $\mathrm{B}$ cells, dendritic cells, monocytes, eosinophils and lower expression on neutrophils, but not expressed on CD34+ haemopoietic cells [18-21].

The early CAMPATH-1 antibodies, rat IgM (CAMPATH-1M) and rat IgG (CAMPATH-1G) were remarkably lytic with human complement. CAMPATH-1G and the humanized form CAMPATH-1H (alemtuzumab), also bind to human IgG Fc receptors and activate antibody-dependent cellular cytotoxicity (ADCC) $[18,22]$. The CAMPATH-1 antibodies have long been used for prevention of GVHD and graft rejection in reduced intensity allogeneic HSCT for haematological malignancies, through the depletion of $\mathrm{T}$ lymphocytes in both donor and recipient [22-24]. Pharmacokinetic studies have shown that alemtuzumab is detectable in the plasma for several weeks after administration, resulting in the depletion of recipient auto-reactive lymphocytes and prevention of GVHD by depletion of donor alloreactive T cells [23, 25], resulting in a low incidence of graft rejection and GVHD, respectively.

\section{The rationale for using alemtuzumab in AA HSCT}

The ideal conditioning regimen in SAA is one that results in sustained engraftment, minimal regimen-related toxicity, the absence of both acute and chronic GVHD, and makes available the potential of HSCT for older patients, who were previously considered ineligible for this treatment. Alemtuzumab is particularly applicable to transplantation for non-malignant diseases, such as SAA, with the aim to induce allograft tolerance.

For patients being transplanted for leukaemia, donor alloreactive $\mathrm{T}$ cells contribute to a fine balance between risks of GVHD and graft-versus leukaemia, but in SAA there is no benefit of any graft-versus- disease effect. However, donor lymphocyte depletion may exacerbate impaired immune reconstitution and potentially increase the risk of viral infections post-transplant. In contrast to HSCT for haematological malignancy, graft failure remains a significant problem in SAA, especially in patients, who have been heavily transfused and are alloimmunized to major and minor HLA antigens. Graft failure occurs in up to $10 \%$ of MSD and $15 \%$ of MUD HSCT patients $[3,6,8,10]$.

In the context of SAA, the knowledge that CD52 is a GPIlinked protein is of prime importance as a relatively large proportion (around 40-50\%) of patients with AA have an associated PNH clone, as detected by highly sensitive flow cytometry. Hence, before considering using alemtuzumab as part of the conditioning regimen for HSCT, if a PNH clone is detected in peripheral blood granulocytes and red cells, recipient $\mathrm{T}$ cells should also be examined for CD52 expression, as deficient expression of CD52 on recipient $\mathrm{T}$ cells may expose the patient to a high risk of rejection when alemtuzumab is used pre-transplant. The presence of a population of recipient $\mathrm{T}$ cells showing absent expression of CD52 would prompt the use of ATG instead of alemtuzumab, although there is no evidence base to define the size of such a population. In our clinical practice, we have used an arbitrary cut off of $>10 \%$ CD52-deficient $\mathrm{T}$ cells.

\section{Early studies using CAMPATH-1 antibodies in SAA HSCT}

The early single centre studies using CAMPATH-1G in the conditioning regimen for MSD HSCT for SAA showed graft failure rates of $24 \%$. The high graft rejection was associated with the initial use of alemtuzumab given both pre- and peri-transplantation in a cohort of 21 patients, in an attempt to achieve maximum GVHD prophylaxis [26]. However, subsequent patients receiving alemtuzumab only pre-transplant showed a reduction in graft rejection to 10-15\%, while maintaining a low incidence of GVHD [27]. Patients had either transient mixed chimerism or persistence of recipient $(<20 \%)$ cells. It was noted that the use of Campath-1G was associated with a high incidence of mixed chimerism which tips the balance away from GVHD. Interestingly, the high survival of early patients with graft failure was in part due to a high incidence of autologous recovery, seen in about $50 \%$ of patients [26].

With the subsequent availability of the humanized form of CAMPATH-1, alemtuzumab, a small single centre study reported stable engraftment and a favourable impact on the incidence and severity of GVHD in patients receiving MUD HSCT for SAA, warranting further investigation of this drug [28].

In another single centre, observational study, the outcomes of 10 consecutive patients treated with alemtuzumab-based GVHD prophylaxis and 14 consecutive patients who received calcineurin inhibitor plus methotrexate-based GVHD prophylaxis were compared [29]. The 


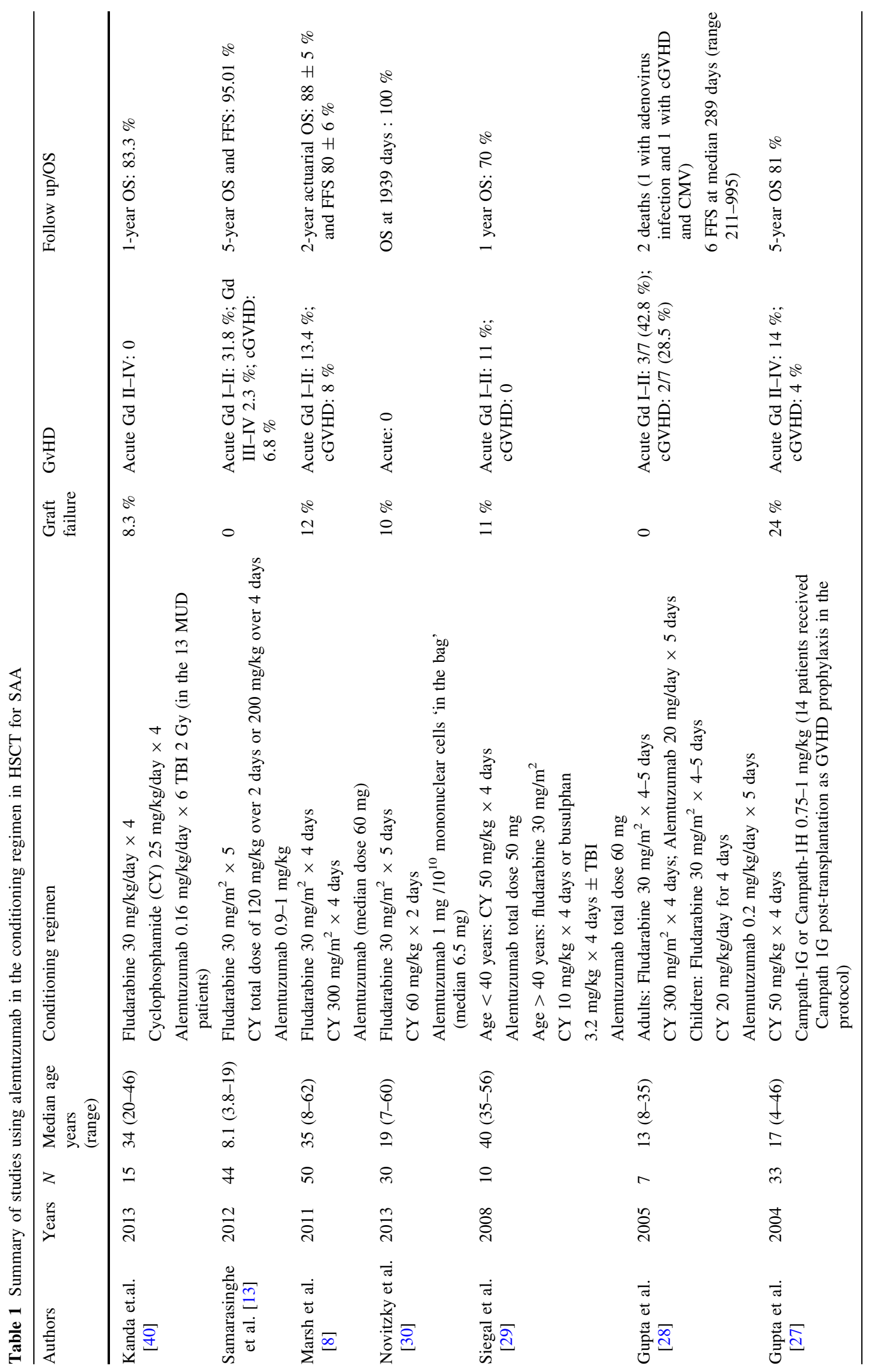


incidence of acute and chronic GVHD was significantly lower in alemtuzumab-treated patients as compared to conventionally treated patients. Engraftment time and rates of graft failure appeared similar in the two groups. Although more alemtuzumab-treated patients developed CMV reactivation none developed CMV disease.

An alternative approach was to use ex vivo $\mathrm{T}$ cell depletion by infusion of stem cell graft that has been T-depleted ex vivo with alemtuzumab, after reduced intensity conditioning with fludarabine and cyclophosphamide. In a series of 30 patients, there were no cases of acute or chronic GVHD, and all patients achieved initial engraftment, but there were 3 cases of late graft failure at 4 and 7 months, and a third following treatment of CMV with ganciclovir [30].
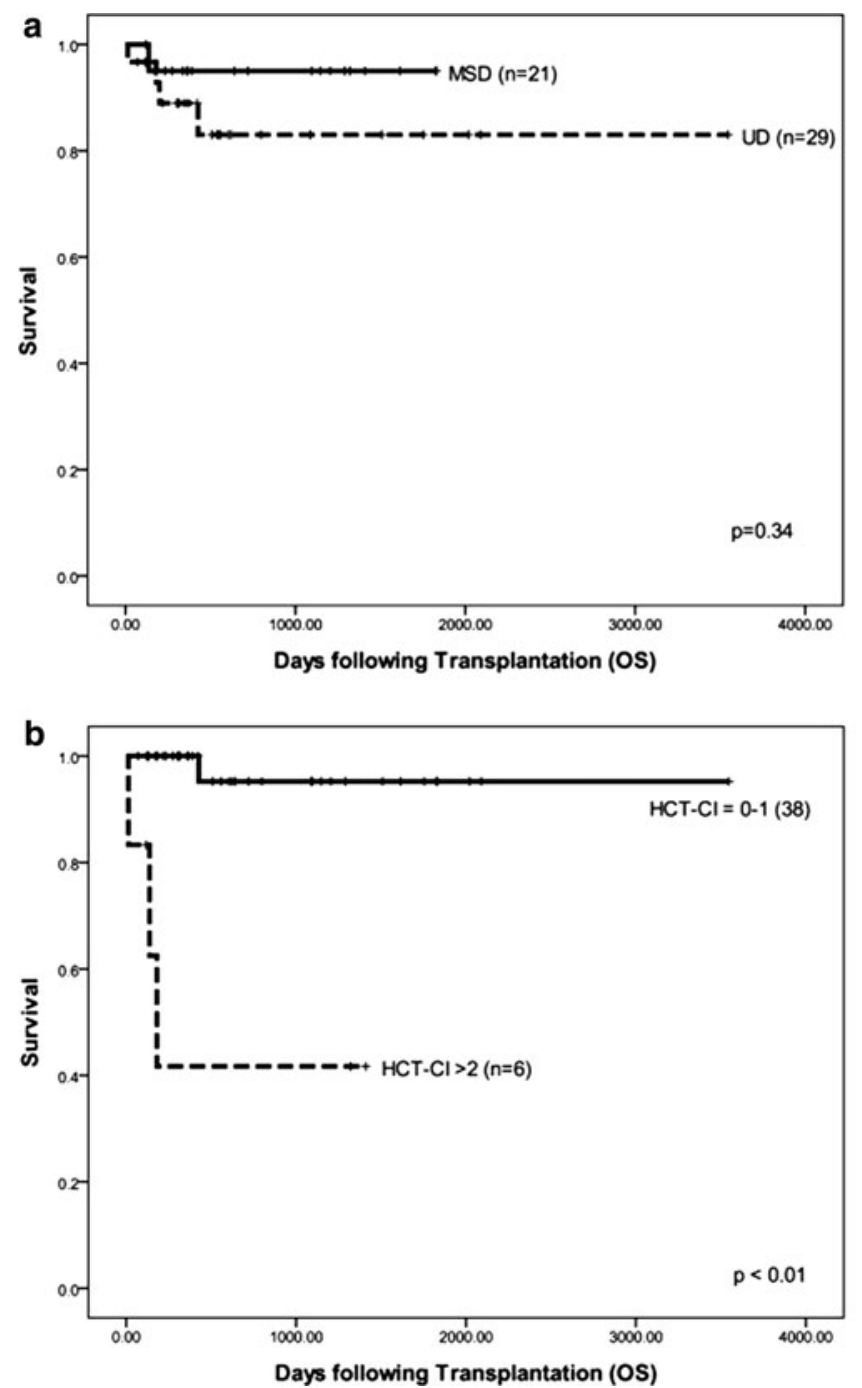

Multicentre, retrospective studies of alemtuzumabbased conditioning in SAA

The novel 'FCC' conditioning regimen of fludarabine $\left(300 \mathrm{mg} / \mathrm{m}^{2}\right)$, cyclophosphamide $\left(300 \mathrm{mg} / \mathrm{m}^{2}\right)$ from days -7 to -4 and alemtuzumab (median dose of $60 \mathrm{mg}$ ) given pre-transplantation from days -7 to -3 , was reported in a retrospective, multicentre study of 50 patients with acquired SAA [8]. The median age was 35 years (range 8-62), and 29 received a transplant from an UD and 21 from an MSD. All UD HSCT patients, apart from 2, were matched for HLA-A, -B, -C, -DRB1, and DQB1 using high-resolution DNA typing. Two patients were transplanted from $9 / 10$ matched unrelated donors and received TBI 2 Gy in addition to FCC conditioning. The 2-year OS
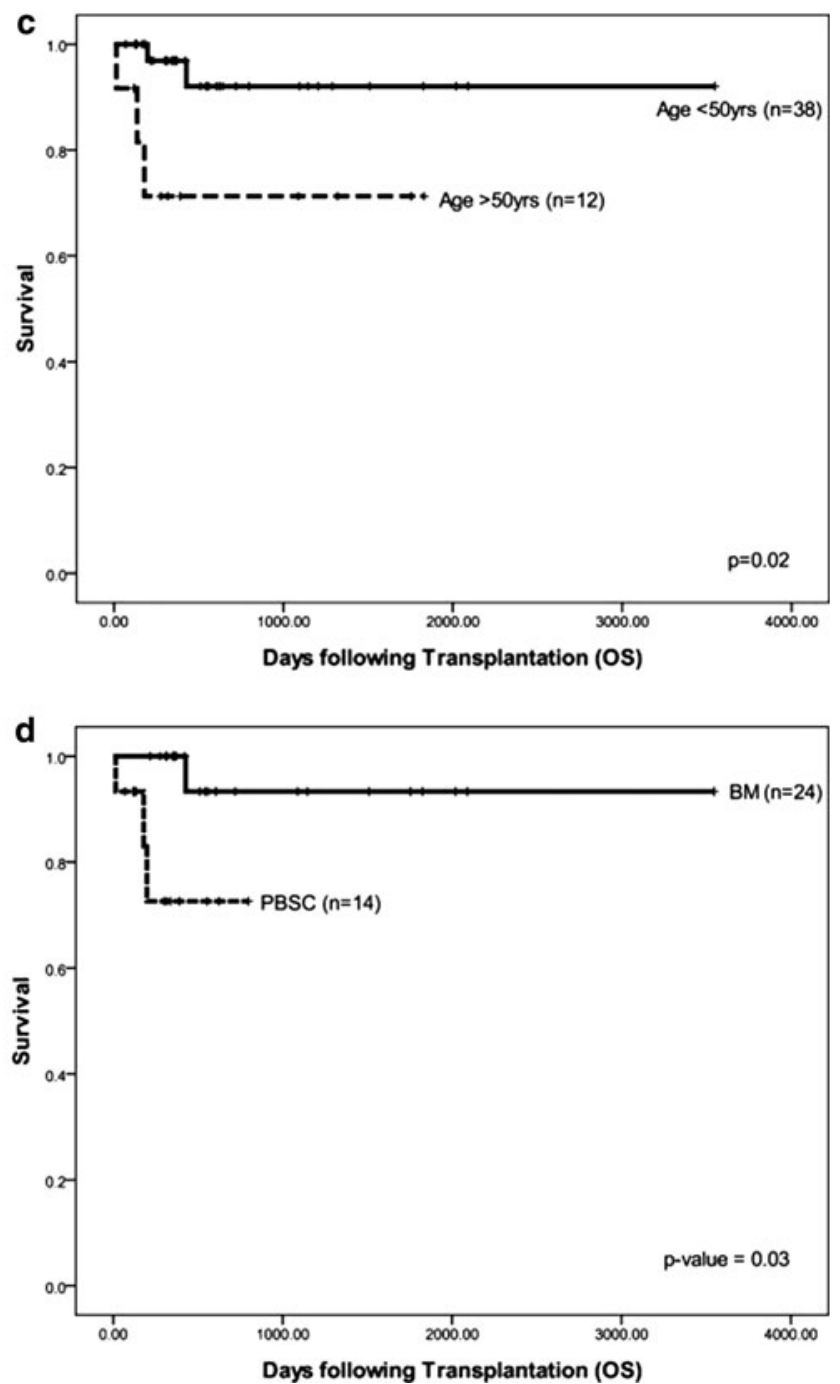

Fig. 1 Overall survival curves following HSCT using FCC conditioning, with stratification for a donor type, b HCT-CI, c age $<50$ years and d stem cell source. With permission of Marsh et al. [8] 
was $95 \%$ for MSD and $83 \%$ for UD HSCT (see Fig. 1a). Cumulative incidence of graft rejection was 9.5 and $14.5 \%$ for MSD and UD HSCT, respectively. The incidence of GVHD was very low: acute GVHD $13.7 \%$ and chronic GVHD $4 \%$ [8].

An important advantage of using alemtuzumab as part of conditioning for MUD HSCT in SAA, was that the addition of low dose ( $2 \mathrm{~Gy}$ ) TBI to aid engraftment, was not required. In contrast, it is common practice to use TBI 2 Gy for fully matched UD HSCT for SAA with ATGbased conditioning, as a previous study reported a graft failure rate of $35 \%$ when only fludarabine, cyclophosphamide and ATG were used in adults with SAA [9, 10].

Mixed donor chimerism, as assessed on unfractionated PB mononuclear cells, occurs frequently after HSCT for SAA [31]. Progressive mixed chimerism carries a high risk of graft rejection, but stable mixed chimerism (SMC) is associated with the absence of chronic GVHD and excellent survival. Assessment of chimerism in PB sub-populations, using the 'FCC' protocol, revealed stable mixed chimerism was frequently seen in $\mathrm{CD} 3+\mathrm{T}$ cells alongside full donor myeloid (CD15) chimerism and normal haematological recovery (see Fig. 2) [8]. Stable mixed T cell chimerism was associated with the absence of chronic GVHD; persistence of mixed $\mathrm{T}$ cell chimerism following the withdrawal of post-graft immunosuppression with CSA, raises the likelihood that a state of tolerance is achieved post-HSCT. Further studies to define T cell subpopulations and immune reconstitution post-HSCT are indicated. For example, as shown in a recent study of reduced intensity HSCT for haematological malignancies using alemtuzumab with fludarabine and busulphan, increased numbers of effector CD4 $\mathrm{T}$ cells and their imbalance relative to regulatory $\mathrm{CD} 4 \mathrm{~T}$ cells, were noted to be a signature of GVHD [32].

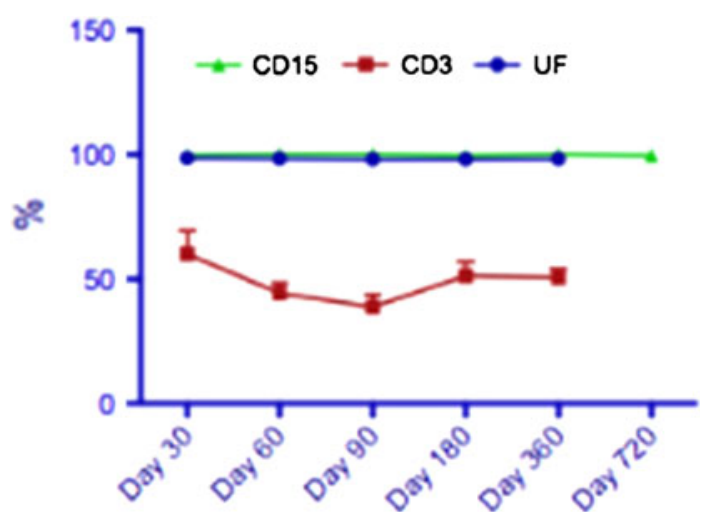

Fig. 2 Chimerism results post-HSCT using FCC conditioning. Representative median $\mathrm{PB}$ chimerism results shown in this figure. $U F$ unfractioned $(n=33), \mathrm{CD} 15(n=16), \mathrm{CD} 3(n=16)$, with permission of Marsh et al. [8]
A second retrospective, multicentre study from the UK, evaluated 44 children who received MUD HSCT, of whom 40 had previously failed IST. The conditioning comprised 'FCC' with a higher dose of CY compared to the study discussed above. BM was used as the stem cell source in $59 \%$ and PBSC in $41 \%$. There were no cases of graft failure and excellent overall and failure-free survival of $95 \%$ at 5 years. Acute GVHD grades I-II occurred in $31.8 \%$ and grades III-IV in one patient $(2.3 \%)$. Chronic GVHD was seen in only 3 patients $(6.8 \%)$, two with limited and one extensive. There was no association between stem cell source and acute or chronic GVHD [13].

A recent small, multicentre study from Japan, examined the effect of alemtuzumab dose in 15 patients with SAA, 12 from UD and $3 \mathrm{MSD}$, who also received fludarabine and $\mathrm{CY}$ $(25 \mathrm{mg} / \mathrm{kg} \times 4)$, and 2 Gy TBI (except in the $3 \mathrm{MSD}$ patients) [33]. The initial dose was $0.2 \mathrm{mg} / \mathrm{kg} / \mathrm{day} \times 6$ (in the first 3 patients) and the next dose was $0.16 \mathrm{mg} / \mathrm{kg} /$ day $\times 6$ in the next 3 . The latter dose was the final dose in the rest of the patients. All patients engrafted, and there was one late graft failure. Mixed T cell chimerism of $>10 \%$ donor cells was observed in $50 \%$ of patients. There were 2 cases of acute GVHD (both Gd I) and 2 cases of chronic GVHD. CMV reactivation occurred in all patients, but there was only one case of $\mathrm{CMV}$ disease. EBV reactivation occurred in 2 patients with no EBV PTLD. Two patients died (late graft failure and sepsis, respectively.

The first study to compare alemtuzumab with ATGbased conditioning regimens in HSCT for SAA has been performed by the British Society for Blood and Marrow Transplantation (BSBMT). In this retrospective, multicentre study of 159 patients, 88 were transplanted from MSD, 65 from MUD and 6 from alternative donors. Alemtuzumab was used in 103 and ATG in 55 patients. The results showed 1, 5 and 10 years OS of 89,85 and $85 \%$, respectively, and there was no difference in 1-year OS between the alemtuzumab and ATG groups [34]. Subsequent analyses have shown better OS for MUD HSCT using alemtuzumab as compared to ATG and no difference for MSD. A lower risk of chronic GVHD was observed with alemtuzumab as compared to ATG (11 vs $26 \%$ ) (J. Marsh, unpublished data 2013). Prospective studies comparing alemtuzumab and ATG-based conditioning are now indicated.

\section{The impact of stem cell dose and source}

A low stem cell dose is associated with an increased risk of graft failure in HSCT for SAA, as initially based on the PB nucleated cell counts [35]. Evaluation of infused BM CD34+ cell dose showed that the risk of graft failure increased significantly with doses of $<2 \times 10^{6} / \mathrm{kg}$ [36]. 
Thus, it is important to ensure an adequate stem cell dose, although data are lacking in terms of the optimal number of $\mathrm{CD} 3+$ cells that is not associated with an increase in the risk of GVHD.

The source of stem cells for SAA HSCT is also important, as studies reported to date, have shown that for ATG-based conditioning regimens, BM is the preferred source as compared to PBSC; the use of PBSC results in a higher incidence of chronic GVHD and worse OS [6, 37, 38]. One of the concerns for MUD HSCT has been the poor quality of bone marrow harvests in some instances, resulting in low stem cell dose harvested. The use of PBSC can enable a higher and at least adequate cell dose in most cases. Using alemtuzumab-based conditioning, the use of PBSC enables an adequate stem cell collection without increasing the risk of chronic GVHD; in that study, there was worse 2-year OS with PBSC as compared to BM, but no difference in failure-free survival [8]. However, the study was too small to compare the results of MSD with MUD HSCT in terms of stem cell source, and further studies on larger number of patients are required.

\section{Infections}

There are concerns about the risk of viral infections associated with the use of alemtuzumab. Some studies have reported a higher incidence of viral infections, including CMV and EBV, following the use of alemtuzumab-based conditioning regimens, when compared with ATG and methotrexate $[39,40]$. However, no increased incidence of CMV, EBV or adenovirus infection or reactivation was reported in the retrospective multicentre adult and pediatric studies reported above [8, 13]. Nevertheless, a regular surveillance program for these viruses should be employed post-HSCT.

\section{Outcomes in older patients}

HSCT for SAA is less successful in older patients. Using conventional high dose $\mathrm{CY}$ and ATG conditioning regimen for MSD HSCT, OS falls to $50 \%$ for people aged 40-50 years [11, 12]. A higher incidence of GVHD and organ toxicity may contribute to worse survival outcomes in older patients. Using the FCC protocol (see Fig. 1c), in the small sub-group of patients aged $>50$ years (range 50-62), 2-year OS was $71 \%$ [8] Thus, the low toxicity of the regimen, may allow older patients, who have previously failed IST treatment, to be considered for HSCT, although larger studies are indicated to explore this further. This study also reported for the first time, the importance of assessing co-morbidities pre-transplant (see Fig. 1b), and this should now be evaluated further in the selection of older patients for HSCT.

\section{Conclusions}

Alemtuzumab in combination with fludarabine and cyclophosphamide (FCC) HSCT for acquired AA is associated with a very low incidence of chronic GvHD and excellent OS, without undue increase in graft failure and infections. Alemtuzumab-based reduced intensity conditioning regimens may be considered in older patients $>50$ years. Prospective randomized trials comparing alemtuzumab and ATG-based conditioning are needed both in matched sibling and unrelated donor setting to confirm the retrospective data and to better understand the potential state of tolerance post-HSCT seen with alemtuzumab.

\section{References}

1. Passweg JR, Aljurf M. Treatment and hematopoietic SCT in aplastic anemia. Bone Marrow Transpl. 2013;48:161.

2. Marsh JCW, Ball SE, Cavenagh J, et al. British Committee for Standards in Haematology (BCSH). Guidelines for the diagnosis and management of aplastic anaemia. Br J Haematol. 2009; $147: 43-70$

3. Konopacki J, Porcher R, Robin M, et al. Long-term follow up after allogeneic stem cell transplantation in patients with severe aplastic anemia after cyclophosphamide plus antithymocyte globulin conditioning. Haematologica. 2012;97:710-6.

4. Champlin RE, Perez W, Passweg J, et al. Bone marrow transplantation for severe aplastic anemia: a randomized controlled study of conditioning regimens. Blood. 2007;109:4582-5.

5. Maury S, Bacigalupo A, Anderlini P, et al. Improved outcome of patients older than 30 years receiving HLA-identical sibling hematopoietic stem cell transplantation for severe acquired aplastic anemia using fludarabine-based conditioning: a comparison with conventional conditioning regimen. Haematologica. 2009;94:1312-5.

6. Bacigalupo A, Socié G, Schrezenmeier H, Aplastic Anemia Working Party of the European Group for Blood and Marrow Transplantation (WPSAA-EBMT), et al. Bone marrow versus peripheral blood sibling transplants in acquired aplastic anemia: survival advantage for marrow in all age groups. Haematologica. 2012;97:1142-8.

7. Viollier R, Socie G, Tichelli A, Working party aplastic anaemia (WPSAA) of the European Group for Blood and Marrow Transplantation (EBMT), et al. Recent improvement in outcome of unrelated donor transplantation for aplastic anaemia. Bone Marrow Transpl. 2007;41:45-50.

8. Marsh JC, Gupta V, Lim Z, et al. Alemtuzumab with fludarabine and cyclophosphamide reduces chronic graft-versus-host disease after allogeneic stem cell transplantation for acquired aplastic anemia. Blood. 2011;118:2351-7.

9. Bacigalupo A, Locatelli F, Dini G, Severe Aplastic Anemia Working Party of the European Group for Blood and Marrow 
Transplantation (SAA WP-EBMT), et al. Fludarabine, cyclophosphamide and anti-thymocyte globulin for alternative donor transplants in acquired severe aplastic anemia: a report from the EBMT-SAA Working Party. Bone Marrow Transpl. 2005;36: 947-50.

10. Bacigalupo A, Socie G, Lanino E, Severe Aplastic Anemia Working Party of the European Group for Blood and Marrow Transplantation, et al. Fludarabine, cyclophosphamide, antithymocyte globulin, with or without low dose total body irradiation, for alternative donor transplants, in acquired severe aplastic anemia: a retrospective study from the EBMT-SAA working party. Haematologica. 2010;95:976-82.

11. Sangiolo D, Storb R, Deeg HJ, et al. Outcome of allogeneic hematopoietic cell transplantation from HLA-identical siblings for severe aplastic anemia in patients over 40 years of age. Biol Blood Marrow Transpl. 2010;16:1411-8.

12. Gupta V, Eapen M, Brazauskas R, et al. Impact of age on outcomes after transplantation for acquired aplastic anemia using HLA-identical sibling donors. Haematologica. 2010;95:2119-25.

13. Samarasinghe S, Steward C, Hiwarkar P, et al. Excellent outcome of matched unrelated donor transplantation in paediatric aplastic anaemia following failure with immunosuppressive therapy: a United Kingdom multicentre retrospective experience. $\mathrm{Br} \mathrm{J}$ Haematol. 2012;157:339-46.

14. Kennedy-Nasser A, Leuknig K, Mahiajan A, et al. Comparable outcomes of matched-related and alternative donor stem cell transplantation for pediatric severe aplastic anemia. Biol Blood Marrow Transpl. 2006;12:1277-84.

15. Yagasaki H, Takahashi Y, Hama A, et al. Comparison of matched-sibling donor BMT and unrelated donor BMT in children and adolescent with acquired severe aplastic anaemia. Bone Marrow Transpl. 2010;45:1508-13.

16. Sanders JE, Hoffmeister PA, Woolfrey AE, et al. Thyroid function following hematopoeitic cell transplantation in children: 30 years experience. Blood. 2009;113:306-8.

17. Sanders JE, Woolfry AE, Carpenter PA, et al. Late effects among pediatric patients followed for nearly 4 decades after transplantation for severe aplastic anemia. Blood. 2011;18:1421-8.

18. Hale G, Cobbold S, Novtzky N, et al. CAMPATH-1 antibodies in stem-cell transplantation. Cytotherapy. 2001;3(3):145-64.

19. Buggins A, Mufti GJ, Salisbury J, et al. Peripheral blood but not tissue dendritic cells express CD52 and are depleted by treatment with alemtuzumab. Blood. 2002;100(5):1715-20.

20. Ambrose LR, More A-S, Warrens AN. Neutrophils express CD52 and exhibit complement-mediated lysis in the presence of alemtuzumab. Blood. 2009;114:3052-5.

21. Gilleece MH, Dexter TM. Effect of Campath-1H antibody on human hematopoietic progenitors in vitro. Blood. 1993;82: 807-12.

22. Dyer MJ, Hale G, Hayhoe FG, Waldmann H. Effects of CAMPATH-1 antibodies in vivo in patients with lymphoid malignancies: influence of antibody isotype. Blood. 1989;73:1431-9.

23. Morris EC, Rebello P, Thomson KJ, et al. Pharmacokinetics of alemtuzumab used for in vivo and in vitro T-cell depletion in allogeneic transplantations: relevance for early adoptive immunotherapy and infectious complications. Blood. 2003;102:404-6.

24. Ho AY, Pagliuca A, Kenyon M, et al. Reduced intensity allogeneic hematopoietic stem cell transplantation for myelodysplastic syndrome and acute myeloid leukemia with multilineage dysplasia using fludarabine, busulphan, and alemtuzumab (FBC) conditioning. Blood. 2004;104:1616-23.

25. Rebello P, Cwynarski K, Varughese M, Eades A, Apperley JF, Hale G. Pharmacokinetics of CAMPATH-1H in BMT patients. Cytotherapy. 2001;3:261-2617.
26. Hamblin M, Marsh JC, Lawler M, et al. Campath-1G in vivo confers a low incidence of graft versus-host disease associated with a high incidence of mixed chimaerism after bone marrow transplantation for severe aplastic anaemia using HLA-identical sibling donors. Bone Marrow Transpl. 1996;17:819-24.

27. Gupta V, Ball SE, Yi QL, et al. Favourable effect on acute and chronic graft-versus-host disease with cyclophosphamide and in vivo anti-CD52 monoclonal antibodies for marrow transplantation from HLA-identical sibling donors for acquired aplastic anemia. Biol Blood Marrow Transpl. 2004;10:867-76.

28. Gupta V, Ball SE, Sage D, et al. Marrow transplants from matched unrelated donors for aplastic anaemia using alemtuzumab, fludarabine and cyclophosphamide based conditioning. Bone Marrow Transpl. 2005;35:467-71.

29. Siegal D, Xu W, Sutherland R, et al. Graft-versus-host disease following marrow transplantation for aplastic anemia: different impact of two GVHD prevention strategies. Bone Marrow Transpl. 2008;42:51-6.

30. Novitzky N, Thomas V, du Toit C. Conditioning with purine analogs leads to good engraftment rates of immunodepleted grafts for aplastic anemia. Biol Blood Marrow Transpl. 2013;19:584-8.

31. Lawler M, McCann SR, Marsh JC, et al. Serial chimerism analyses indicate that mixed haemopoietic chimerism influences the probability of graft rejection and disease recurrence following allogeneic stem cell transplantation (SCT) for severe aplastic anaemia (SAA): indication for routine assessment of chimerism post SCT for SAA. Br J Haematol. 2009;144: 933-45.

32. Matthews K, Lim Z, Pearce L, et al. Imbalance of effector and regulatory $\mathrm{CD} 4 \mathrm{~T}$ cells is associated with graft-versus-host disease after hematopoietic stem cell transplantation using a reduced intensity conditioning regimen and alemtuzumab. Haematologica. 2009;94:956-66.

33. Kanda Y, Oshima K, Kako S, et al. In vivo T-cell depletion with alemtuzumab in allogeneic hematopoietic stem cell transplantation: combined results of two studies on aplastic anemia and HLA-mismatched haploidentical transplantation. Am J Hematol. 2013;88:294-300.

34. Marsh JCW, Pearce RM, Koh MB, et al. National, retrospective, multi-centre comparison of alemtuzumab- versus ATG-based conditioning regimens in hematopoietic stem cell transplantation for aplastic anemia: a study from the British Society for Blood and Marrow Transplantation (BSBMT) (CTCR 09-03). Blood (ASH annual meeting abstracts). 2011;118:52.

35. McCann SR, Bacigalupo A, Gluckman E, et al. Graft rejection and second bone marrow transplants for acquired aplastic anaemia: a report from the aplastic anaemia working party of the European bone marrow transplant group. Bone Marrow Transpl. 1994;13:233-7.

36. Islam MS, Anoop P, Datta-Nemdharry P, et al. Implications of CD34+ cell dose on clinical and haematological outcome of alloSCT for acquired aplastic anaemia. Bone Marrow Transpl. 2010;45(5):886-94.

37. Schrezenmeier H, Passweg J, Marsh JCW, et al. Worse outcome and more chronic GVHD with peripheral blood progenitor cells than bone marrow in HLA-matched sibling donor transplants for young patients with severe acquired aplastic anemia. Blood. 2007;110:1397-400.

38. Eapen M, Le-Rademacher J, Antin J, et al. Effect of stem cell source on outcomes after adult unrelated donor transplantation in severe aplastic anemia. Blood. 2011;118:2618-21.

39. Park SH, Choi SM, Lee DG, Choi JH, et al. Infectious complications associated with alemtuzumab use for allogeneic 
hematopoietic stem cell transplantation: comparison with antithymocyte globulin. Transpl Infect Dis. 2009;11:413-23.

40. Lamba R, Carrum G, Myers GD, et al. Cytomegalovirus (CMV) infections and CMV-specific cellular immune reconstitution following reduced intensity conditioning allogeneic stem cell transplantation with alemtuzumab. Bone Marrow Transpl. 2005;36(9):797-802. 